\title{
A Highly Sensitive Determination of Vitamin D in Supplements Using Graphene-Nafion Nanocomposite
}

\author{
Abdulhadee Yakoh ${ }^{1}$, Orawon Chailapakul1,2and Weena Siangproh ${ }^{3^{*}}$ \\ ${ }^{1}$ Electrochemistry and Optical Spectroscopy Center of Excellence (EOSCE), Department of \\ Chemistry, Faculty of Science, Chulalongkorn University, 254 Phayathai Road, Patumwan, Bangkok \\ 10330, Thailand \\ ${ }^{2}$ Center for Petroleum, Petrochemicals and Advanced Materials, Chulalongkorn University, 254 \\ Phayathai Road, Pathumwan, Bangkok 10330, Thailand \\ ${ }^{3}$ Department of Chemistry, Faculty of Science, Srinakharinwirot University, Sukhumvit 23, Wattana, \\ Bangkok 10110 \\ Corresponding author's e-mail address:weena@g.swu.ac.th
}

\begin{abstract}
In this work, we describe an antifouling strategy for highly sensitive determination of fat-soluble vitamin D based on graphene/nafion nanocomposite. Graphene based screen-printable ink was used to fabricate graphene/nafion nanocomposite with good reproducibility. Besides the sensitivity improvement, a graphene/nafion nanocomposite exhibited a lower background current in comparison with carbon and graphene screen-printed electrode (SPE), which resulted in a larger signal-tobackground (S/B) ratio. In addition, under the synergistic effect of graphene and nafion, a sensitive, reusable, and simple electrochemical sensor was developed for evaluating the total level of vitamin D. Under the optimal conditions, the sensor demonstrates excellent linear relation in the range of $0.5-30$ $\mu \mathrm{g} \mathrm{mL} \mathrm{L}^{-1}$ with a correlation coefficient of 0.9916 . The limit of detection (LOD) and quantitation (LOQ) from the calculation are found to be 68.8 and $229.6 \mathrm{ng} \mathrm{mL}^{-1}$, respectively. Furthermore, to evaluate the reproducibility with different graphene/nafion composite electrode $(n=10)$ in term of the relative standard deviation (\%RSD), three concentrations of vitamin D were determined. \%RSD of each concentration were found to be less than $7 \%$, suggesting the good reliability and reproducibility of the developed sensor for vitamin D. In final, diverse pharmaceutical drugs and dietary supplements were analyzed, which results obtained from the developed sensor were in good agreement with the LC-MS/MS method.
\end{abstract}

Keywords: Vitamin D, Graphene, Nafion, Anti-fouling, Electroanalysis 